\title{
Statistical Features of the Quiet Sun in EUV
}

A. Pauluhn

INTEC HTA Bern and Inst. of Astronomy, ETH Zürich, Switzerland

S. K. Solanki

Max-Planck-Institut für Aeronomie, Katlenburg-Lindau, Germany

I. Rüedi

Physikalisch Meteorologisches Observatorium, World Radiation Center Davos, Switzerland

E. Landi and U. Schühle

Max-Planck-Institut für Aeronomie, Katlenburg-Lindau, Germany

\begin{abstract}
Frequency distributions of the intensities of EUV emission lines in the quiet Sun have in the past usually been modelled using two Gaussians. Here we test this and other distribution functions against observed distributions with exceptional statistics. The data were obtained in a number of spectral lines observed with CDS and SUMER. We show that the frequency distribution of the radiance is best modelled by a lognormal distribution. The fact that the radiance distribution of the quiet Sun including the network and the intranetwork is better reproduced by a single lognormal distribution function than by two Gaussians suggests that the same heating processes are acting in both types of features.
\end{abstract}

\section{Introduction}

Several studies have shown that the radiance of the quiet Sun in EUV emission lines follows a distribution with a pronounced peak and a long tail extending to high intensities (Huber et al. 1974; Reeves et al. 1976; Lemaire et al. 1997; Bocchialini et al. 1997; Griffiths et al. 1999; Dammasch et al. 1999). The skewed form of the distribution precludes a fit with a single Gaussian. Fits consisting of two distributions introduce a natural distinction between brighter and darker parts of the solar atmosphere, usually equated with the network and cell interiors, while a single distribution suggests that there is no principle distinction between various parts of the quiet Sun, at least not a simple one. We tested five statistical models of probability functions against the data: a Rayleigh distribution, a Maxwellian distribution, a superposition of two Gaussian distributions, a lognormal distribution $\left(\rho(x)=\frac{N_{0}}{x} e^{-\frac{(\ln (x)-\mu)^{2}}{2 \sigma^{2}}}\right)$, and a superposition of a lognormal and a Gaussian distribution. Which of these approaches provides a better 
description can only be decided on the basis of a large amount of data, i.e. distributions of extremely high statistical significance. Besides, we compare distributions obtained with the two different instruments, SUMER and CDS. How these distributions change with formation temperature of the lines is described in Pauluhn et al. 2000.

\section{Observations and data}

The observations have been made during the $\mathrm{SOHO}$ intercalibration programme through March 1996 and June 1998. The large set of data consists of simultaneous radiance measurements of CDS and SUMER of common targets near solar disk centre devoid of any notable activity (Pauluhn et al. 1999). The spectral lines recorded during these measurements were $\mathrm{He}$ I at $584 \AA$ and the two $\mathrm{Mg} \mathrm{x}$ lines at $609 \AA$ and $624 \AA$. SUMER additionally observed in Ne VIII $770 \AA$ and in Nv $1238 \AA$. Our sample of lines with good statistics thus covers formation temperatures ranging from $2 \times 10^{4} \mathrm{~K}$ to $1 \times 10^{6} \mathrm{~K}$, i.e. the chromosphere, transition region and corona. Each measurement consisted of a raster scan over an extended area. After the instrumental corrections and the radiometric calibration, the solar radiances were determined by integration over Gaussian fits to the line profiles and suitably binned before forming histograms.

\section{Intensity statistics of the quiet Sun}

The lognormal and double Gaussian fits to the observed distributions are plotted in Fig. 1. The upper row shows the results for the He I $584 \AA$ line $\left(T=2 \times 10^{4}\right.$ $\mathrm{K}$, left two plots: SUMER, right two plots: CDS). The lower row shows the same but for the coronal line $\mathrm{Mg} \times 609 \AA\left(T=1 \times 10^{6} \mathrm{~K}\right)$. Based on the analysis of the fits to all the lines observed we can summarize the following points:

1) The fits based on the Maxwell and Rayleigh distribution functions are of relatively poor quality.

2) The double Gaussian fit (6 free parameters) captures more than $80 \%$ of the intensity but is unable to account for the prominent tail of the distributions.

3) The lognormal distribution (3 free parameters) alone covers around $90 \%$ of the intensity in all temperature ranges. It is able to represent the tails of the distributions quite accurately.

4) The combined model of a lognormal and a Gaussian (6 free parameters) provides the best fit for most lines, in particular those formed at coronal temperatures. However, the distinction between the two components is arbitrary and not particularly stable, so that the improvement of some of the fits is mainly a consequence of the increased number of free parameters.

For the coronal lines, the lognormal fits are significantly better for the SUMER than for the CDS data. We found that this is due to the difference in spatial resolution between the two instruments by degrading suitable SUMER data (raster scans) to CDS resolution. However, only for the lines formed at higher temperature do the distributions change with resolution. For the chromospheric He I $584 \AA$ line there was no improvement at higher resolution. It therefore seems that the spatial elements contributing to the EUV emissions of 
HeI $584 \AA$ :
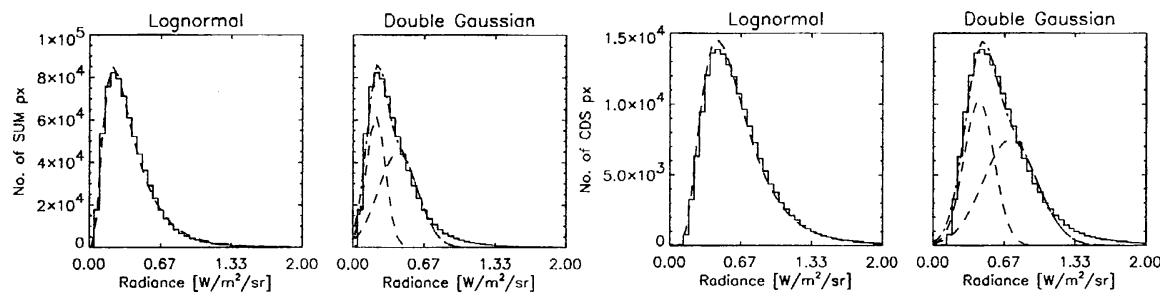

$\operatorname{Mg} \times 609 \AA ̊$
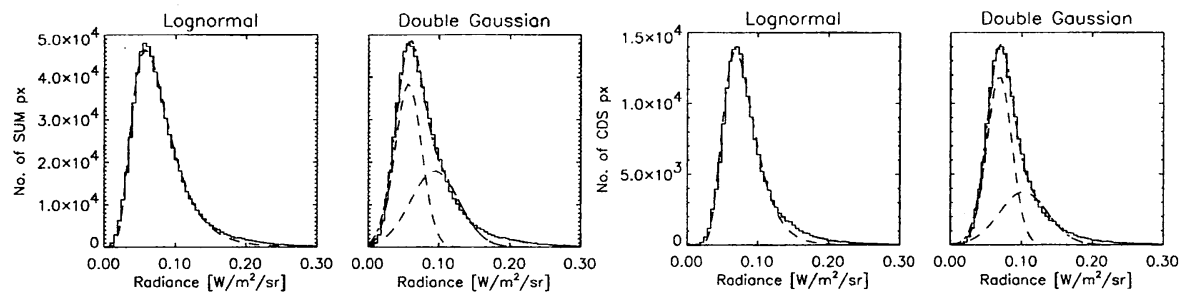

Figure 1. Upper row: Frequencies of occurrence at He I $584 \AA$, fitted with the 2 different models, left 2 plots: SUMER, right 2 plots: CDS. Lower row: The same for the $\mathrm{Mg}$ x $609 \AA$ line.

lower temperature regions can be resolved, whereas for coronal lines the available SUMER resolution is not sufficient, and the size of these heating elements lies at or below SUMER's resolution of $1^{\prime \prime}$. This confirms that the relevant scales of coronal heating lie at or below the spatial resolution of SUMER. The fact that in most cases the lognormal distribution is to be preferred to a double Gaussian provides support for the idea that basically the same heating processes are acting in the network and in cell interiors.

\section{References}

Bocchialini K., Vial J.-C., \& Einaudi G., 1997, $5^{\text {th }}$ SOHO Worksh., Oslo, ESA SP-404, 211

Dammasch I.E., Hassler D.M., Curdt W., \& Wilhelm K., 1999, Space Sci. Rev. 87, 161

Gallagher P.T., Phillips K.J.H., Harra-Murnion L.K., \& Keenan F.P., 1998, A\&A 335, 733

Griffiths N.W., Fisher G.H., Woods D.T., \& Siegmund O.H.W., 1999, ApJ 512, 992

Huber M.C.E., Foukal P., Noyes R.W. et al., 1974, ApJ 194, L115

Lemaire P., Wilhelm K., Curdt W. et al., 1997, Sol. Phys. 170/1, 105

Pauluhn A., Rüedi I., Solanki S.K. et al., 1999, Appl. Optics 38, No. 34, 7035

Pauluhn A., Solanki S.K., Rüedi I., et al., 2000, to appear in A\&A.

Reeves E.M., Vernazza J.E, Withbroe G.L., 1976, Phil. Trans. R. Soc. London, Ser. A 281,319 